\title{
Marie-Luise Recker
}

\section{Das Parlament in der westdeutschen Kanzlerdemokratie}

„Der Bundeskanzler bestimmt die Richtlinien der Politik und trägt dafür die Verantwortung." Mit dieser lapidaren Aussage in Art. 65 GG ist eine entscheidende Grundlage der „Kanzlerdemokratie“ umschrieben, die als Kennzeichen des bundesrepublikanischen Regierungssystems ${ }^{1}$ gilt. In der Tat hatten sich die Mitglieder des Parlamentarischen Rates zum Ziel gesetzt, eine Verfassungsordnung für den entstehenden westdeutschen Staat zu schaffen, in der im Vergleich zu ihrer Weimarer Vorgängerin die Gewichte zwischen den Verfassungsorganen neu austariert und damit die strukturellen Schwächen der ersten deutschen Demokratie - sofern sie auf der Verfassung beruhten - möglichst beseitigt werden sollten. Dies betraf ${ }^{2}$ zum einen die Reduzierung der Präsidialgewalt und die Beschränkung der Befugnisse des Bundespräsidenten auf im wesentlichen repräsentative Funktionen, zum anderen die Stärkung der Position des Regierungschefs gegenüber dem Präsidenten, dem Kabinett und dem Parlament und nicht zuletzt die engere Bindung der Regierungsfraktionen an die von ihr getragene Regierung, ein Vorsatz, der ebenfalls der Schaffung einer starken Regierungsgewalt zugute kam.

Die Herausbildung und Stabilisierung der Kanzlerstellung zur eindeutigen und dominanten Machtposition des bundesrepublikanischen Regierungssystems basierte einerseits auf den einschlägigen Bestimmungen des Grundgesetzes, erfolgte andererseits ebenso durch die Art, wie Adenauer - gleichsam stilbildend - dieses Amt ausfüllte und seinen Aktionsradius ausbaute. Das Kabinett, über die Richtlinienkompetenz an die vom Kanzler vorgegebene politische Linie gebunden, wurde vom Regierungschef an der kurzen Leine geführt ${ }^{3}$ und fand zu keinem ge-

1 Vgl. zu diesem Begriff noch immer Anselm Doering-Manteuffel, Strukturmerkmale der Kanzlerdemokratie, in: Der Staat 30 (1991) 1-18, sowie Karlheinz Niclauß, Kanzlerdemokratie. Bonner Regierungspraxis von Konrad Adenauer bis Helmut Kohl (Stuttgart 1988). 2 Vgl. hierzu Udo Wengst, Staatsaufbau und Regierungspraxis 1948-1953. Zur Geschichte der Verfassungsorgane der Bundesrepublik Deutschland (Düsseldorf 1984) 246-301, sowie Volker Otto, Das Staatsverständnis des Parlamentarischen Rates. Ein Beitrag zur Entstehungsgeschichte des Grundgesetzes für die Bundesrepublik Deutschland (Düsseldorf 1971) 122-137.

${ }^{3}$ Dies lassen die mittlerweile für die Jahre 1949 bis 1959 publizierten Kabinettsprotokolle deutlich erkennen. 
schlossenen Auftreten gegen ihn zusammen. Die Minister, auf Vorschlag des Kanzlers vom Bundespräsidenten ernannt, konnten ihre Ressorts nur innerhalb der vom Kanzler bestimmten politischen Generallinie selbständig und unter eigener Verantwortung leiten. Mit der Geschäftsordnung der Bundesregierung, von Adenauer penibel redigiert ${ }^{4}$, suchte er dies zusätzlich zu untermauern. Als zentrale Stütze erwies sich schließlich das Bundeskanzleramt, das Adenauer zur Schaltzentrale der Regierungspolitik auszubauen und als Kontroll- und Steuerungsinstrument gegenüber dem Kabinett, den einzelnen Ministerien, dem Parlament und der Öffentlichkeit zu etablieren verstand. Nicht zuletzt wurde die starke Stellung des Kanzlers dadurch untermauert, daß er zunächst wichtige Politikbereiche ${ }^{5}$ - so die auswärtigen Beziehungen und die Verteidigungspolitik selbst leitete. Auch wenn seine Machtposition nicht überzeichnet werden sollte -, die sich aus dem Grundgesetz ergebende starke Stellung des Kanzlers und sein autoritärer Regierungsstil haben Adenauers Dominanz als Regierungschef ausgemacht. Hieran knüpften seine Nachfolger an.

Stellung und Gewicht des Bundestages müssen im Rahmen dieser „Kanzlerdemokratie" ausgelotet werden. In den Verfassungsberatungen hatte es keine großen Kontroversen um seine Rolle und Kompetenzen gegeben, vielmehr hatten sich die Verfassungsväter im wesentlichen ,an das allgemeine seit Jahrzehnten in Deutschland geltende Parlamentsrecht" ${ }^{\text {" }}$ angeschlossen und dem Parlament die zentrale Rolle in der Gesetzgebung, einen starken Einfluß auf die Regierungsbildung und schließlich Mitwirkungsrechte bei der Wahl des Bundespräsidenten zugeschrieben. Gegenüber der für seinen Weimarer Vorgänger geltenden Regelung wurde seine Auflösung erschwert und damit seine Stellung gegenüber Bundeskanzler und Bundespräsident gefestigt, gleichzeitig jedoch seine Befugnisse, eine einmal gewählte Regierung zu stürzen, beschnitten. Erst in Relation zu den Bestimmungen für die anderen Verfassungsorgane erhielt die Volksvertretung ihren spezifischen Ort in der bundesrepublikanischen Verfassungsordnung.

Nicht nur in bezug auf seine verfassungsmäßige Stellung, sondern auch in organisatorischer Hinsicht knüpfte der Bundestag in vielen Bereichen an seinen Weimarer Vorgänger an. Dies gilt für das Selbstverständnis als Arbeitsparlament, das seine vornehmste Aufgabe in der Gesetzgebung sah und die entsprechenden Entwürfe vor allem in seinen Ausschüssen ausführlich diskutierte und umgestaltete. Dies gilt für seine Geschäftsordnung7, die seine Arbeitsweise, ja, generell den

${ }_{4} \mathrm{Vgl}$. Rudolf Morsey, Adenauers Anteil an der Formulierung der Geschäftsordnung der Bundesregierung von 1951, in: Klaus Grupp, Michael Ronellenfitsch (Hrsg.), Planung - Recht - Rechtsschutz. Festschrift für Willy Blümel zum 70. Geburtstag am 6. Januar 1999 (Berlin 1998) 385-402.

${ }^{5}$ Für die ersten Jahre bedeutsam war ebenfalls die Rücksichtnahme auf die Souveränitätsbeschränkungen nach dem Besatzungsstatut, wo Adenauer seine Kontakte zur alliierten "Oberregierung“ auf dem Petersberg zur Untermauerung der Kanzlerstellung nutzte.

6 So der Abgeordnete Walter (CDU) im Hauptausschuß des Parlamentarischen Rates am 11. 11. 1948, vgl. Parlamentarischer Rat, Verhandlungen des Hauptausschusses (o.O. o. J.) 2. 7 Am 20. September 1949 verständigte sich der Bundestag darauf, bis auf weiteres nach der nur in wenigen Punkten geänderten Geschäftsordnung des Reichstages zu verfahren. Vgl. 
parlamentarischen Alltag bestimmte und die dezidiert an die im Reichstag praktizierte Geschäftsordnung anknüpfte. Dies gilt auch für Sitzordnung, parlamentarisches Zeremoniell und Sitzungsablauf8, wo ebenfalls überkommene Muster weitgehend übernommen wurden. Angesichts der tiefen Brüche in der deutschen Geschichte wurde die Tradition eines bis 1871, ja, bis 1848 zurückreichenden Parlamentarismus als positiver Anknüpfungspunkt und Legitimationsstrang angesehen. Anleihen an die parlamentarischen Bräuche in anderen europäischen Staaten waren dagegen eher selten.

Auch die parteipolitische Zusammensetzung des ersten Deutschen Bundestages gemahnte an Weimarer Zeiten; immerhin zogen acht Fraktionen und weitere Gruppen sowie Fraktionslose in die Pädagogische Akademie am Rheinufer ein. Diese politische Buntscheckigkeit, die die Bundestagswahl 1949 dem Anschein nach zu einem Urnengang auf halbem Weg zwischen Weimar und Bonn ${ }^{9}$ werden ließ, beeinflußte auch die Atmosphäre im Bundeshaus. Der Einzug von Mitgliedern der KPD, der Deutschen Konservativen/Deutschen Rechtspartei und der Wirtschaftlichen Aufbauvereinigung, von Parlamentariern wie Max Reimann und Heinz Renner, Fritz Dorls und Alfred Loritz, Wolfgang Hedler und Herwart Mießner prägten den Debattenstil dort nachhaltig. Polemische Zwischenrufe, Störungen der Redner, ja, selbst tumultarische Szenen ${ }^{10}$ und der Ausschluß von Parlamentariern von den nächsten Plenarsitzungen waren an der Tagesordnung. Der Bundestagspräsident sah sich immer wieder vor Schwierigkeiten, die Kontrahenten zur Ordnung zu rufen und seine Autorität durchzusetzen. Mit den Wahlen 1953, nach denen nur noch fünf Fraktionen den Sprung in den Bundestag schafften, versachlichte sich der Ton. Diese Konzentration auf wenige Fraktionen setzte sich in den folgenden Wahlen fort; sie war die deutlichste Abkehr von Weimar.

Die Marginalisierung der kleineren Parteien auf der linken und der rechten Seite des politischen Spektrums war nicht nur eine Folge des 1953 geänderten Wahlrechts, sondern ebenso Quintessenz der Integrationskraft der anderen Parteien. Der Siegeszug des Volksparteikonzepts ${ }^{11}$ prägte das Parteiensystem der

Fritz Sänger (Hrsg.), Die Volksvertretung. Handbuch des Deutschen Bundestages (Stuttgart 1949) 70-99. Auch die zum 1. Januar 1952 in Kraft getretene neue Geschäftsordnung orientierte sich in vielen Teilen an ihrer Vorgängerin. Vgl. BGBl. 1952 II 389.

${ }^{8} \mathrm{Vgl}$. hierzu Marie-Luise Recker, Westminster als Modell? Der Deutsche Bundestag und das britische Regierungssystem, in: Gerbard A. Ritter, Peter Wende (Hrsg.), Rivalität und Partnerschaft. Studien zu den deutsch-britischen Beziehungen (Paderborn 1999) 313-335, sowie dies., „Es braucht nicht niederreißende Polemik, sondern aufbauende Tat". Zur Parlamentskultur der Bundesrepublik Deutschland, in: Jahrbuch des Historischen Kollegs 2002, 67-88. 9 So die These in Jürgen W. Falter, Kontinuität und Neubeginn. Die Bundestagswahl 1949 zwischen Weimar und Bonn, in: PVS 22 (1981) 236-263.

10 Als Beispiele sei auf die Reaktion auf Kurt Schumachers Zwischenruf am 24. 11. 1949 vom „Kanzler der Alliierten“ sowie auf die Tumulte nach einem Verweis des Abgeordneten Hedler aus dem Plenarsaal verwiesen. Vgl. Peter Merseburger, Der schwierige Deutsche. Kurt Schumacher. Eine Biographie (Stuttgart 1995) 459-464, sowie Norbert Frei, Vergangenheitspolitik. Die Anfänge der Bundesrepublik und die NS-Vergangenheit (München 1996) 315-319. 11 Vgl. hierzu Dorothee Buchbaas, Die Volkspartei. Programmatische Entwicklung der CDU 1950-1973 (Düsseldorf 1981), sowie hinsichtlich des Konzepts der Sammlung des bür- 
Bundesrepublik nachhaltig; im Bundestag erlangten somit diejenigen die politische Hegemonie, die sich diesem Konzept am deutlichsten annäherten. Die Sammlung des bürgerlichen Lagers hinter den Unionsparteien, der sich auf Dauer nur die FDP entziehen konnte, bedeutete die Überwindung der parteipolitischen Zersplitterung, die den Weimarer Reichstag geprägt und die Koalitionsbildung dort erschwert hatte. Trotz Abschleifung der programmatischen Profile und ungeachtet deutlicher Flügelbildung - etwa bei CDU und FDP - gelang den Parteien die Integration ihrer Wähler und Mitglieder, was der politischen Stabilität und der Arbeitsfähigkeit des Bundestages zugute kam.

Die scharfe Konfrontation zwischen den beiden größten Parteien, die sich im Bundestag als Regierungs- und Oppositionsfraktion gegenüberstanden, begünstigte dieses Sammlungskonzept. Der - zumindest nach dem Parteiprogramm scharfe Antagonismus von Union und Sozialdemokratie, etwa in der Wirtschaftsoder in der Außen- und Deutschlandpolitik, prägte den Argumentations- und Debattenstil auch im Bundestag und ließ den kleineren Parteien wenig Raum für die eigene Profilierung. Weder als Vertreter regionaler (Bayernpartei, Deutsche Partei) noch als Advokat sozialer Interessen (GB/BHE) vermochten sie wählerwirksame eigenständige Akzente zu setzen. Die Einbindung zumindest einiger dieser kleineren Parteien in die Regierungskoalition tat ein übriges, deren Profilierungsmöglichkeiten zu begrenzen. Zudem wurden die politischen Erfolge der Bundesregierung, ob „Wirtschaftswunder" und Ausbau des Sozialstaats, ob Westbindung und Europäische Integration, eher dem Kanzler und seiner Partei gutgeschrieben, was dessen Konzept der Sammlung des bürgerlichen Lagers um die Unionsachse entgegenkam. Die parteipolitische Konstellation im Bundestag unterschied sich somit grundlegend von der im Reichstag der Weimarer Republik.

Die Zusammenarbeit unter den Bundestagsfraktionen entsprach jedoch nur bedingt dem gängigen Gegenüber von Regierung und Opposition. Die KPD auf der linken und die Deutsche Rechtspartei bzw. Nationale Rechte auf der rechten Seite der Sitzordnung des Bundestages blieben im parlamentarischen Alltag ausgegrenzt $^{12}$. Ihre Initiativen fanden keine Unterstützung bei den anderen Fraktionen, in der Ausschußarbeit wurden sie marginalisiert, im parlamentarischen Procedere galten sie als nicht gleichrangig, und durch die neue Geschäftsordnung 1952 verloren sie wichtige parlamentarische Rechte, so daß ihre Aktionsfähigkeit weiter reduziert wurde. Die anderen Parlamentarier suchten keine Gemeinsamkeiten mit ihnen, im Gegenteil, jede Unterstützung durch diese Gruppierungen wurde brüsk abgewiesen.

Die Fraktionen diesseits dieses extremistischen Randes kooperierten im parlamentarischen Alltag auf unterschiedliche Weise ${ }^{13}$. Dies bedeutete nicht, daß politische Differenzen nicht offen ausgetragen wurden; dies galt sowohl für die mit

gerlichen Lagers Frank Bösch, Die Adenauer-CDU. Gründung, Aufstieg und Krise einer Erfolgspartei 1945-1969 (Stuttgart 2001).

12 Vgl. für Details Wolfgang Kralewski, Karlheinz Neunreither, Oppositionelles Verhalten im ersten Deutschen Bundestag 1949-1953 (Köln, Opladen 1963) 36, 50.

13 Vgl. ebd. 46-50. 
scharfem rhetorischen Schwert geführten Debatten um außenpolitische Fragen als auch beispielsweise für die Konkurrenz der drei bayerischen Parteien CSU, Bayernpartei und Wirtschaftliche Aufbauvereinigung, die sich in wirtschafts- und sozialpolitischen Angelegenheiten heftige Positionskämpfe lieferten. Dies hieß jedoch wohl, daß die jeweiligen Fraktionen je nach Sachthema punktuell zusammenarbeiteten, etwa bei Gesetzesinitiativen, bei Änderungsanträgen, bei parlamentarischen Anfragen oder in Abstimmungen. Zumindest für das bürgerliche Lager kann dies als eine Vorstufe zur Sammlungspolitik der Union gesehen werden, da hier die sachliche Übereinstimmung der Regierungsfraktionen mit einzelnen Oppositionsparteien (Zentrum, WAV, BP) deutlich wurde. Eine analoge Kooperation unter den Oppositionsfraktionen gab es nicht; nicht allein die Ausgrenzung der kommunistischen Abgeordneten verhinderte eine solche Zusammenarbeit, auch jenseits der KPD fehlte der Impuls zu einer (selbst punktuellen) Kooperation. Die mangelnde Hoffnung auf spätere Zusammenarbeit in einer Regierung sprach aus diesem Verhalten ebenso wie das Beharren auf Eigenständigkeit seitens der kleineren Fraktionen, die argwöhnten, andernfalls zum Anhängsel der beiden Großen zu werden. Dieses wechselnde, uneinheitliche Stimmverhalten begünstigte die Regierungsparteien, deren parlamentarische Mehrheit im Einzelfall verbreitert wurde. Im zweiten Deutschen Bundestag kam dies zunächst nicht zum Tragen, da die SPD nun die einzige Oppositionspartei war; erst mit dem Ausbrechen von Teilen von GB/BHE und FDP aus der Regierungskoalition 1955/56 wurde das Abstimmungsverhalten dort wieder uneinheitlicher, wobei auch nun eine Kooperation innerhalb der Opposition die Ausnahme blieb.

Unter den Oppositionsparteien entfaltete die SPD bei weitem die größte Initiativkraft; dies galt für Anfragen, Anträge wie Gesetzesvorlagen ${ }^{14}$ gleichermaßen. Mit diesen Initiativen wollten die sozialdemokratischen Parlamentarier nicht nur ihren Wählern ihren beharrlichen Einsatz für die eigenen programmatischen Ziele dokumentieren, sondern nicht minder ihre Stellung als Widerpart der Regierung und als Regierung im Wartestand dokumentieren. So beabsichtigten sie, etwa über Große Anfragen ${ }^{15}$, die Versäumnisse der Bundesregierung aufzuzeigen und diese unter Zugzwang zu setzen, möglichst bald auf diese Vorstöße zu reagieren. Durch eigene Gesetzesinitiativen sollte zudem in den anstehenden Ausschußdebatten dem dort bereits vorliegenden Regierungsentwurf ein eigenes Konzept entgegengesetzt und so versucht werden, das künftige Gesetz mitzugestalten. In der ersten Legislaturperiode legte die SPD-Fraktion mehr als 50 Gesetzesentwürfe vor und stellte mehr als 200 Anträge. Auch in der zweiten Wahlperiode konnte sie mit 90 Gesetzesentwürfen und 150 Anträgen eine stolze Bilanz aufweisen. In den nächsten Legislaturperioden ${ }^{16}$ nahmen diese Zahlen leicht $a b$, doch blieb die Initiativ-

$14 \mathrm{Vgl}$. ebd. 56-63.

15 Vgl. für Beispiele: Die SPD-Fraktion im Deutschen Bundestag. Sitzungsprotokolle 1949-1957, bearb. v. Petra Weber, Erster Halbband (Düsseldorf 1993) XCI-XCIII. In der 1. Wahlperiode stellte die SPD-Fraktion 77, in der 2. Wahlperiode 55 Große Anfragen.

16 Angaben nach: Die SPD-Fraktion im Deutschen Bundestag. Sitzungsprotokolle 19571961, bearb. v. Wolfgang Hölscher (Düsseldorf 1993) XLV, sowie: Die SPD-Fraktion im 
tätigkeit mit über 50 selbständigen Gesetzesentwürfen in der dritten und über 70 in der vierten Wahlperiode vergleichsweise hoch. Der Schwerpunkt dieser Vorstöße lag - dem eigenen Selbstverständnis entsprechend - im sozialpolitischen und innenpolitischen Bereich, wo sie sich als Wahrer sozialer Belange und als Verfassungspartei profilieren wollte. Zugute kam ihr hierbei, daß sie sich auf den Parteiapparat, insbesondere auf die jeweiligen Fachausschüsse beim Parteivorstand, sowie auf SPD-geführte Landesregierungen und deren Ministerialbürokratie stützen konnte.

Diese Möglichkeiten standen den anderen Oppositionsfraktionen nicht zu Gebote. Auch die KPD versuchte, über parlamentarische Initiativen ${ }^{17}$ auf sich aufmerksam zu machen und die eigenen politischen Vorstellungen auf diese Weise zu verdeutlichen. Allerdings hatten diese Vorschläge in der Regel vornehmlich Propagandacharakter und zielten eher auf die eigene Anhängerschaft als auf die Kollegen in den anderen Fraktionen. Zudem waren die KPD-Abgeordneten im parlamentarischen Alltag weitgehend ausgegrenzt, verloren mit der neuen $\mathrm{Ge}$ schäftsordnung von Anfang 1952 ihren Fraktionsstatus und damit gewisse Initiativrechte, so daß es ihnen nicht gelang, die parlamentarische Arbeit mir ihren Vorstößen zu prägen. Selbst die Zentrumspartei versuchte anfangs, mit der regen Initiativtätigkeit von SPD und KPD mitzuhalten, mußte jedoch bald einsehen, daß sie hierzu weder personell noch materiell in der Lage war. Für die anderen Oppositionsfraktionen galt dies in noch größerem Maße.

Hatte sich unter den Oppositionsparteien keine mehr als punktuelle Zusammenarbeit entwickelt, so mußten die Koalitionsfraktionen schon im Interesse der Stützung der Bundesregierung darauf bedacht sein, sich gegenseitig abzustimmen und mit Kanzler und Kabinett zu kooperieren. Trotz immer wieder aufkommender Klagen gelang dies im großen und ganzen auch. Allerdings war gerade für die kleineren Koalitionspartner in manchem Fall der Reiz groß, ihre Wahlchancen dadurch zu erhöhen, daß man sich von der Partei des Regierungschefs absetzte und die eigene Wählerklientel in besonderem Maße ansprach. So entwickelten auch die FDP- und die DP-Fraktion ${ }^{18}$ eine rege Initiativtätigkeit, die sich an derjenigen von Zentrum und Bayernpartei durchaus messen konnte. Selbst die Landesgruppe der $\mathrm{CSU}^{19}$ versuchte, sich auf diese Weise als eigenständige Kraft innerhalb der Koalition zu präsentieren und in bestimmten Sachfragen auch gegenüber den bayerischen Konkurrenten BP und WAV Flagge zu zeigen. Allerdings erwies sich dies nie als Sprengsatz für die Regierungskoalition, gerade in der ersten Legislaturperiode sahen Kanzler und Fraktionsführung der Union ein solches Verhalten offenbar als Zugeständnis an das langsame und für die

Deutschen Bundestag. Sitzungsprotokolle 1961-1963, bearb. v. Heinrich Potthoff (Düsseldorf 1993) CIII.

17 Vgl. Kralewski, Neunreither, Oppositionelles Verhalten 35.

18 Ebd. Die SPD brachte im Ersten Deutschen Bundestag 485, die KPD 180 Initiativen ein. Die FDP stand mit 136 Initiativen an dritter, die DP mit 103 Initiativen an siebter Stelle.

19 Vgl. ebd. $44 \mathrm{f}$. 
kleineren Partner heikle Zusammenwachsen innerhalb der bürgerlichen Koalition. In den folgenden Legislaturperioden verlief die Kooperation geräuschloser, wenn es auch immer wieder Profilierungsbemühungen gerade der kleineren Partner gab.

Spezielle Gremien oder Foren der Abstimmung zwischen den Koalitionsparteien selbst und mit dem Kabinett wurden nicht installiert. Ein noch im September 1949 geschaffener Koordinierungsausschuß ${ }^{20}$ blieb praktisch ohne Belang. Auch nach den folgenden Regierungsbildungen blieb es bei informellen Absprachen; erst 1961 einigten sich CDU, CSU und FDP darauf, einen Koalitionsausschuß, bestehend aus den Fraktionsvorsitzenden, deren Stellvertretern und den Parlamentarischen Geschäftsführern, zu installieren, in dem anstehende Probleme und Differenzen besprochen werden sollten. So blieb es in den 1950er Jahren vor allem den Fraktionsspitzen vorbehalten, diese Zusammenarbeit sicherzustellen.

Heinrich von Brentano, der erste Vorsitzende ${ }^{21}$ der Unionsfraktion, war allerdings kein selbstbewußter, dem Kanzler ebenbürtiger Gegenpart, der die Fraktion mit fester Hand geführt hätte. Er verstand sich eher als Moderator, als Verbindungsglied zwischen den Koalitionsabgeordneten und dem Kabinett, letztlich auch als Stütze und Rückhalt der Regierung. Auch wenn er die unzulängliche Information und die Ignorierung der Fraktion seitens der Bundesregierung immer wieder kritisierte, setzte er doch auf Arrangement und Anpassung und beugte sich der Autorität des Kanzlers. Trotz gelegentlichen Aufbegehrens und der Sorge, daß "die Zusammenarbeit zwischen dem Kabinett und der Fraktion trotz meiner unermüdlichen Bemühungen " 22 zu wünschen übrig ließe, schwenkte er letztlich auf die Linie des Regierungschefs ein.

Eigentlicher Motor der Kooperation und Mittler zwischen den Unionsabgeordneten und dem Kabinett war eher Heinrich Krone, ab August 1951 Parlamentarischer Geschäftsführer der Fraktion und seit 1955, nach dem Wechsel von Brentanos an die Spitze des Auswärtigen Amtes, selbst Fraktionsvorsitzender ${ }^{23}$. Durch seine politische Statur, seine ausgleichende Kraft und Mittlerfunktion wuchs er in eine parlamentarische Schlüsselstellung hinein; durch die enge Abstimmung mit Adenauers engstem Mitarbeiter, Globke, kam ihm eine entscheidende Funktion im Kräftedreieck von Bundestagsfraktion, Partei und Regierung zu. Besser als von Brentano verstand er es, Unstimmigkeiten und Spannungen im Vorfeld auszuloten, „die Fraktion dem Kanzler gegenüber in Ordnung zu hal-

20 Vgl. Die CDU/CSU-Fraktion im Deutschen Bundestag. Sitzungsprotokolle 1949-1953, bearb. v. Helge Heidemeyer (Düsseldorf 1998) XCVI.

21 Für wenige Sitzungen, nämlich bis zu seiner Wahl zum Bundeskanzler, war Adenauer Vorsitzender der Unionsfraktion, am 30. September 1949 folgte ihm von Brentano im Vorsitz.

22 Schreiben von Brentanos an Adenauer vom 16.6. 1951, abgedruckt in Arnulf Baring (Hrsg.), Sehr verehrter Herr Bundeskanzler! Heinrich von Brentano im Briefwechsel mit Konrad Adenauer 1949-1964 (Hamburg 1974) 64-70, hier 66.

${ }^{23}$ Vgl. Heinrich Krone, Tagebücher. Erster Band: 1945-1961, bearb. v. Hans-Otto Kleinmann (Düsseldorf 1995), vgl. insbes. die Einleitung Kleinmanns. 
ten" 24 sowie die Kooperation der Fraktion mit Kanzler und Kabinett, aber auch der Koalitionsfraktionen untereinander, weitgehend geräuschlos zu gestalten.

Die kleineren Koalitionsparteien waren nicht in erster Linie durch ihre Fraktionsvorsitzenden, sondern zumindest in gleichem Maße durch ihre Parteivorsitzenden und Bundesminister in die Koordination der Koalitionsfraktionen einbezogen. In den Sitzungen der FDP-Fraktion war es vor allem Franz Blücher, Parteichef, Marshallplan-Minister und Vizekanzler, der die Politik der Bundesregierung erläuterte und versuchte ${ }^{25}$, die Unterstützung der Fraktion zu gewinnen. Ähnliches galt für Heinrich Hellwege in der DP- und für Theodor Oberländer in der BHE-Fraktion. Den Fraktionsvorsitzenden kam demgegenüber eher die Funktion zu, die unterschiedlichen Interessen in den eigenen Reihen auszugleichen und eine einheitliche Linie zu erarbeiten. Die konfliktreichste Zeit in der $\mathrm{Zu}$ sammenarbeit der Koalitionsfraktionen waren wohl die Jahre, in denen Thomas Dehler, Fraktionschef und Bundesvorsitzender der FDP, sich zu einem streitbaren Widerpart Adenauers entwickelte ${ }^{26}$ und Blücher, durch den Wechsel im Parteivorsitz geschwächt, dies nicht auszugleichen vermochte. In der zweiten Legislaturperiode unternahm der Kanzler sogar den Versuch, durch die Ernennung von Robert Tillmanns (CDU), Herrmann Schäfer (FDP), Waldemar Kraft (GB/BHE) und Franz Josef Strauß (CSU) zu Bundesministern für besondere Aufgaben im Kabinett spezielle Verbindungsleute zu den Koalitionsfraktionen (bzw. zur CSULandesgruppe) zu installieren, doch erwies sich dies als wenig erfolgreich und wurde 1955/56 beendet.

Die informelle Kooperation zwischen der Regierung und den Koalitionsfraktionen ${ }^{27}$ etwa im Ältestenrat des Bundestages, über die Zuziehung der Fraktionsspitze zu Kabinettssitzungen, über Gespräche im kleinen Kreis oder über die Präsenz des Kanzlers in Fraktions- und Fraktionsvorstandssitzungen funktionierte relativ gut und unspektakulär. Die Fraktionsvorsitzenden, Mitglieder des Fraktionsvorstandes oder auch einzelne Abgeordnete wurden mehr oder weniger regelmäßig zu Kabinettssitzungen zugezogen ${ }^{28}$, was zwar das Einvernehmen zwischen Fraktion und Regierung verbesserte, aber auch auf Widerspruch stieß. Adenauer erblickte in den Koalitionsfraktionen vornehmlich ein Hilfsorgan der Regierung, eine Sichtweise, die diese nicht hinnehmen wollten und die sie durch Betonung der eigenen Standpunkte zu konterkarieren suchten. Dennoch ging dies nie soweit, daß ein Bruch mit der Regierung und eine mögliche parlamentarische Niederlage in Kauf genommen worden wären.

24 So Kurt Georg Kiesinger in: Hans Buchbeim (Hrsg.), Konrad Adenauer und der Deutsche Bundestag (Bonn 1986) 47.

25 Dies lassen die überlieferten Sitzungsprotokolle der FDP-Fraktion erkennen. Vgl. Archiv des Deutschen Liberalismus, Gummersbach, A 40.

26 Vgl. hierzu Udo Wengst, Thomas Dehler 1897-1967. Eine politische Biographie (München 1997) 235-279.

27 Hierauf weist Rudolf Morsey, Konrad Adenauer und der Deutsche Bundestag, in: Buchheim, Adenauer 14-40, hier 20, hin.

28 Vgl. CDU/CSU-Fraktion im Deutschen Bundestag 1949-1953, XCI f. 
Für die Unionsfraktion traf auch nicht zu, was bereits für die SPD-Parlamentarier konstatiert wurde, nämlich die Kooperation mit und Stütze auf den Parteiapparat. Zwar ist auch für die Sozialdemokratie die alte These vom politischen Übergewicht des Parteivorstandes über die Bundestagsfraktion zu modifizieren und auf die kooperative Zusammenarbeit und personelle Verflechtung zwischen beiden Gremien ${ }^{29} \mathrm{zu}$ verweisen. Dennoch war für die Oppositionsarbeit der Fraktion der wissenschaftliche Apparat des Parteivorstandes unverzichtbar. Für die Union galt letzteres nicht, da sie im Ernstfall auf die Zuarbeit der Ministerialbürokratie in Bonn zurückgreifen konnte.

Die Bundespartei CDU, organisatorisch wie politisch eher schwach, war kein Partner oder Gegenpart zur Fraktion. Zwar ist auch für die Unionsparteien eine deutliche personelle Überschneidung der Führungszirkel von Partei und Fraktion $^{30}$ zu konstatieren, so daß die Koordination zwischen beiden Organen relativ reibungslos war, doch konnte die Bundespartei, vom Kanzleramt aus geführt und ohne nennenswerten Apparat, kein eigenständiger politischer Akteur werden. Selbst wenn Parteigremien in Entscheidungen von Kabinett und Fraktion miteinbezogen wurden - als Beispiel sei auf die Kindergeldgesetzgebung in der zweiten Legislaturperiode ${ }^{31}$ verwiesen, wo die entsprechenden Gesetzesvorschläge monatelang zwischen Familienministerium, Fraktion und Partei hin- und herwechselten, ohne daß in den zentralen Streitpunkten ein Kompromiß gefunden werden konnte -, war dies eher die Ausnahme als die Regel. Für die kleineren Koalitionsparteien galt die weitgehende Ausklammerung von Parteigremien für die Parlamentsarbeit noch viel mehr; deren organisatorisch schwacher Parteiapparat konnte den Fraktionen keine Hilfestellung leisten.

In der Gesetzgebungsarbeit standen dem Bundestag durchaus Gestaltungsspielräume offen, die er auch zu nutzen suchte. Dies galt für die Innen- und Rechtspolitik ebenso wie für Wirtschaftsordnung und Sozialaufbau, die angesichts der "Gründungskrise“32 der Bundesrepublik zunächst im Vordergrund der parlamentarischen Diskussionen standen. Kanzler und Kabinett waren bereit, hier den Regierungsfraktionen in einem gewissen Umfang ein eigenes Wirkungsfeld zuzugestehen, zumal so Kritik an der Dominanz der Exekutive abgefangen und Gruppeninteressen besser eingebunden werden konnten. Zugute kam dem Parlament als politischem Akteur, daß auch die SPD als größte Oppositionsfraktion hier ihre aktive Mitgestaltung anbot, sofern die zu verabschiedenden Gesetze ihrem Selbstanspruch als soziale Reformpartei entsprachen.

29 Dies betont Petra Weber in der Einleitung zu SPD-Fraktion im Deutschen Bundestag 1949-1957, LV-LVIII.

30 So auch Helge Heidemeyer in der Einleitung zu CDU/CSU-Fraktion im Deutschen Bundestag 1949-1953, LXXXIII.

31 Vgl. hierzu die Unterlagen im Parlamentsarchiv des Deutschen Bundestages, Bonn, Gesetzesdokumentation 2. Wahlperiode, II 99.

32 Hans Günter Hockerts, Integration der Gesellschaft - Gründungskrise und Sozialpolitik in der frühen Bundesrepublik, in: Manfred Funke (Hrsg.), Entscheidung für den Westen. Vom Besatzungsstatut zur Souveränität der Bundesrepublik 1949-1955 (Bonn 1988) 39-57. 
Die Ausschüsse waren die eigentliche Arbeitsebene des Parlaments. Hier trafen sich politisch Gleichgesinnte, was die Tendenz begünstigte, über Parteigrenzen hinweg zu kooperieren und sich in besonderer Weise der Belange der eigenen „Klientel“ anzunehmen. Dies galt für den Ausschuß für Sozialpolitik ebenso wie für den Heimatvertriebenenausschuß, für den Wohnungsbauausschuß ebenso wie für den Verkehrsausschuß, für den Wirtschaftsausschuß ebenso wie für den Landwirtschaftsausschuß. Die Konsequenz dieser Konstellation war, daß die Kooperationsbereitschaft zwischen Regierungs- und Oppositionsvertretern groß und breite Abstimmungsmehrheiten - auch in zentralen Gesetzesvorhaben - eher die Regel als die Ausnahme waren. Gerade in der ersten Legislaturperiode war die Zusammenarbeit zwischen den Sozialpolitikern von Union und SPD in den entsprechenden Ausschüssen relativ eng, während sich in den folgenden Jahren ${ }^{33}$ programmatisch-politische Unterschiede eher in den Vordergrund schoben und somit Kontroversen und Konflikte zunahmen. Aber selbst in einem für den Kanzler so zentralen Aktionsfeld wie der Wehrgesetzgebung konnten sich Koalitionsfraktionen und Opposition darauf verständigen, entsprechende Regierungsentwürfe zurückzuweisen und aus den Ausschüssen heraus eigene Vorlagen ${ }^{34} \mathrm{zu}$ erarbeiten, die dann im Plenum eine breite Unterstützung fanden. Insgesamt bleibt festzuhalten, daß die Ausschußarbeit von einer eher kooperativen Atmosphäre geprägt war; „sie festigte den Grundkonsens der Bonner Demokratie, der in den Anfangsjahren der Bundesrepublik noch keineswegs selbstverständlich war" 35 .

Eine neue Situation entstand, wenn die in den Ausschüssen erarbeiteten Vorlagen in zweiter und dritter Lesung im Plenum behandelt wurden. Nun mußte der zuvor erarbeitete Entwurf vor den Gesamtfraktionen bestehen, wo nun auch andere Interessen, Standpunkte und Maximen artikuliert wurden. Mehr oder weniger lange Einigungsverhandlungen zwischen den unterschiedlichen Anschauungen, auch zwischen den Koalitionsfraktionen, Abänderungsanträge in der zweiten und dritten Lesung oder auch ein nicht geschlossenes Stimmverhalten konnten die Folge sein. Während sich die SPD-Fraktion relativ geschlossen zeigte, die Spannweite ihrer Flügel auch letztlich nicht so groß war wie bei den Christdemokraten, mußten insbesondere die Koalitionsfraktionen lernen, die unterschiedlichen Interessen in ihren Reihen zu bündeln und Kompromißlinien zu erarbeiten. Vor allem über die Ausschußvorsitzenden und deren Stellvertreter - wenn diese aus den Reihen der eigenen Fraktion kamen - oder - wenn dies nicht der Fall war über die für die jeweiligen Ausschüsse benannten Obleute wurde der Informa-

33 Als Beispiele sei für die 1. Legislaturperiode auf das 1. Wohnungsbaugesetz, das Rentenneuordnungsgesetz oder das Montanmitbestimmungsgesetz verwiesen, während die SPDFraktion z. B. dem Betriebsverfassungs- sowie dem Lastenausgleichsgesetz ihre Zustimmung verweigerte. In der 2. Wahlperiode gab es keinen Konsens etwa in der Familien- und der Wohnungsbaupolitik, während die sozialdemokratischen Abgeordneten wiederum dem Rentengesetz 1957 zustimmten.

34 Vgl. zur sog. "großen Wehrkoalition“ 1955/56 Hans Eblert, Christian Greiner, Georg Meyer, Bruno Tho $\beta$, Die NATO-Option (München 1993) 430-560.

${ }_{35}$ Petra Weber, Einleitung, in: SPD-Fraktion im Deutschen Bundestag 1949-1957, LXXXIX. 
tionsaustausch zwischen Ausschüssen und Gesamtfraktion ${ }^{36}$ organisiert und die Abstimmung zwischen beiden Gremien schon während der Ausschußberatungen in Gang gehalten. Auch hier erwies sich die erste Legislaturperiode als holprige Wegstrecke; in einer Vielzahl von Abstimmungen gingen Risse durch die Koalitionsfraktionen ${ }^{37}$ oder waren zumindest einzelne Abgeordnete nicht bereit, sich in die Fraktionsdisziplin einzureihen. In den folgenden Jahren gelang die Koordination besser, so daß die Geschlossenheit der Regierungsfraktionen zunahm.

Um die Beratung der Gesetzesvorlagen zu beschleunigen und die Abstimmung zwischen Gesamtfraktion und Ausschußmitgliedern zu optimieren, schuf die Unionsfraktion bereits 1951 fraktionsinterne Arbeitskreise ${ }^{38}$ für konkrete Aufgabengebiete bzw. zu bestimmten Themen, in denen sich interessierte Abgeordnete, Fachleute und Ausschußmitglieder trafen und entsprechende Gesetzesvorhaben vorberieten. In der zweiten Legislaturperiode, als die Fraktion auf fast $250 \mathrm{Köpfe}$ angewachsen war, war diese Untergliederung in Arbeitskreise ein unumgängliches Instrument, um die parlamentarische Arbeit zu organisieren und Gesetzesvorhaben gründlich zu diskutieren. In den Fraktionssitzungen selbst referierten dann die Vorsitzenden und Mitglieder der Arbeitskreise zu den anstehenden Punkten, so daß der Abgleich mit den anderen Standpunkten und Argumenten gesucht werden konnte. Ohne diese Arbeitsteilung und Spezialisierung wären Fraktionssitzungen kaum handhabbar gewesen. Diesem Muster folgte 1953 die SPDFraktion ${ }^{39}$, wenig später auch die FDP-Fraktion, während die kleineren Fraktionen zu einer solchen Binnendifferenzierung personell nicht in der Lage waren. Der zum Teil hochkomplexen Gesetzesmaterie, über die der Bundestag zu befinden hatte, entsprach diese Spezialisierung innerhalb der Fraktionen, die nur auf diese Weise ihren Beratungs- und Entscheidungsprozeß zeitlich und inhaltlich steuern konnten.

Neben seiner Funktion als Gesetzgeber, wo er ein äußerst fleißiger Akteur war, war der Bundestag auch Forum politischer Grundsatzdebatten, insbesondere in außenpolitischen Fragen. Die großen Redeschlachten um Westverträge und Wiedervereinigung, um Wehrbeitrag und Souveränitätsgewinn, um europäische Einigung und deutsch-französische Aussöhnung gehören zu den Höhepunkten der Parlamentsgeschichte. Alle Fraktionen sahen in Debatten zur Außen- und Deutschlandpolitik ein zentrales Forum, um die eigenen Sichtweisen, Handlungsoptionen und Zukunftsperspektiven darzulegen und um Zustimmung zu werben. Außenpolitik war sozusagen die „Königsdisziplin“, in der vor allem die Fraktionsspitzen auftraten, um die eigenen außenpolitischen Ansätze zu entfalten, in der sich aber auch politische Talente als Debattenredner fanden, die sich für Höheres qualifizieren wollten.

36 Vgl. für Einzelheiten: CDU/CSU-Fraktion im Deutschen Bundestag 1949-1953, LVII f. 37 Vgl. hierzu Heinz Markmann, Das Abstimmungsverhalten der Parteifraktionen in deutschen Parlamenten (Meisenheim/Glan 1955) $96 \mathrm{f}$.

$38 \mathrm{Vgl}$. zu diesen Arbeitskreisen: CDU/CSU-Fraktion im Deutschen Bundestag 1949-1953, XLIII-XLVII.

39 Vgl. SPD-Fraktion im Deutschen Bundestag 1949-1957, XLIII. 
In die konkrete Gestaltung der Außenpolitik, insbesondere in Vertragsverhandlungen, war der Bundestag jedoch nicht eingebunden ${ }^{40}$. Hier suchte der Kanzler mit Nachdruck seinen Aktionsradius und seine Kompetenzen zu wahren. Unmut auf allen Seiten der parlamentarischen Bühne über unzureichende Unterrichtung durch die Regierung, über ungebührlichen Zeitdruck und Geheimniskrämerei waren die Folge. Ohne Zweifel wachte Adenauer mit Akribie über seine Kompetenzen und erwartete von den Koalitionsfraktionen die volle Unterstützung für seinen Kurs. Dennoch ist dieses Bild zu wenig differenziert. Auch in außenpolitischen Fragen setzte der Kanzler darauf, einem ausgewählten Kreis von Parlamentariern zumeist aus den Koalitionsfraktionen die zentralen Punkte der anstehenden Abkommen zu erläutern, ihre Unterstützung zu erlangen, sie zu Mittlern gegenüber der Gesamtfraktion zu machen und so die Ratifizierung der Verträge im Parlament sicherzustellen. Ein Mittelweg zwischen der diplomatisch gebotenen Diskretion und Verschwiegenheit und der vertraulichen Einbeziehung von Partei und Fraktion war jedoch nicht leicht zu finden, so daß die Klagen über Informationsdefizite, Zeitdruck und einen unsensiblen Umgang mit der Volksvertretung nicht verstummten.

An der Schnittstelle von Außenpolitik und Parlament lag der Auswärtige Ausschuß. Auch seine Mitglieder ${ }^{41}$ klagten über die unzureichende Unterrichtung durch den Bundeskanzler und (ab 1955) den Außenminister, über unangemessenen Zeitdruck und die Verweigerung der Einsicht in Vertragstexte und andere Dokumente. Auf der anderen Seite sah Adenauer durchaus die Vorteile, die eine Kooperation mit dem Auswärtigen Ausschuß ergab; über ihn konnte sein außenpolitischer Kurs abgesichert und die zügige Ratifizierung der Vertragswerke vorbereitet werden. Versuche, auch die Opposition für eine "bipartisan foreign policy“ zu gewinnen, sind jedoch nicht weit gediehen, die Konzepte beider Parteien lagen zu weit auseinander. Für die Opposition war zudem die Versuchung groß, sich über außenpolitische Themen zu profilieren, und auch die nach wie vor zögerliche Informationspolitik der Bundesregierung war nicht dazu angetan, Vertrauen und Kooperationsbereitschaft der SPD-Abgeordneten zu stärken.

Generell war der Auswärtige Ausschuß nicht in der Lage, sich als wirkungsvoller politischer Akteur neben der Bundesregierung in Szene zu setzen, aus seiner Position als „vorbereitendes Beschlußorgan“ des Plenums auszubrechen und eine "mitentscheidende Kontrolle" der Regierungspolitik auszuüben ${ }^{42}$. Grundsatzdebatten über die Linien deutscher Außenpolitik, über Westintegration und Wieder-

40 Den größten Einfluß auf außenpolitische Abkommen konnte der Bundestag hinsichtlich der „Bindungsklausel“ (Artikel 7/III) des Deutschlandvertrages 1952/54 sowie mit der Präambel zum deutsch-französischen Vertrag 1963 erlangen.

41 Vgl. hierzu generell: Der Auswärtige Ausschuß des Deutschen Bundestages. Sitzungsprotokolle 1949-1953, bearb. v. Wolfgang Hölscher (Düsseldorf 1998) LIX-XCVII.

42 So Wolfgang Hölscher, in: ebd. LXXI. Dies sollte sich auch in der zweiten und dritten Legislaturperiode nicht wesentlich ändern. Vgl. Der Auswärtige Ausschuß des Deutschen Bundestages. Sitzungsprotokolle 1953-1957, bearb. v. Wolfgang Hölscher (Düsseldorf 2002) sowie Der Auswärtige Ausschuß des Deutschen Bundestages 1957-1961, bearb. v. Joachim Wintzer u. Josef Boyer (Düsseldorf 2003). 
vereinigung, Europapolitik und Sicherheitspolitik entwickelten sich dort nur selten, vielmehr standen meist Detailfragen und konkrete Vorhaben im Vordergrund seiner Arbeit. Umstritten war und blieb die Frage, wieweit er sich eigenständig mit außenpolitischen Problemen befassen durfte oder auf die Beratung der vom Plenum an ihn überwiesenen Fragen beschränkt blieb. Auch wenn er in der Praxis ein gewisses „Selbstbefassungsrecht" durchsetzen konnte, zeigt die Tatsache, daß dies umstritten war und blieb, doch die begrenzten Aktionsmöglichkeiten des Ausschusses an.

Auch in Finanzfragen versuchte die Bundesregierung, die Gestaltungsräume des Bundestages einzuschränken. Nach Art.113 GG bedurften Beschlüsse des Bundestages, die die Ausgaben des Haushaltsplanes erhöhen und neue Ausgaben in sich schließen würden, der Zustimmung der Bundesregierung; ein analoger Artikel $(\$ 96)$ wurde auch in die Geschäftsordnung des Bundestages übernommen. Insbesondere Finanzminister Schäffer ${ }^{43}$ setzte darauf, auf diese Weise das Ausgabengebahren des Parlaments steuern und einschränken zu können. Allerdings wehrten sich nicht nur die Oppositionsparteien gegen dieses Ansinnen und verwiesen auf die uneingeschränkte Gesetzgebungsbefugnis des Bundestages. Tatsächlich konnte die Bundesregierung einen Widerspruch gegen ausgabenwirksame Gesetze de facto nicht durchsetzen; die steten Mahnungen Schäffers hinsichtlich des begrenzten Finanzspielraums der Bundesregierung waren zwar eine eindrucksvolle und teils erfolgreiche Drohkulisse, die die Koalitionsfraktionen zu Ausgabendisziplin drängte, doch gegen vom Bundestag beschlossene ausgabenwirksame Gesetze hat die Regierung keinen Einspruch erhoben. Nach einer erfolgreichen Klage der SPD-Fraktion vor dem Bundesverfassungsgericht gegen den entsprechenden Paragraphen der Geschäftsordnung des Bundestages ${ }^{44}$ war dann auch diese Drohung stumpf geworden.

Ein letztes Wort im Gesetzgebungsverfahren hatte der Bundesrat. Auch die Länderkammer hatte eine größere Zahl von Ausschüssen eingesetzt, um so die ihr überwiesenen Vorlagen beraten zu können. Dieses bis ins Detail gehende Mitwirken an der Gesetzgebung stieß bei Bundesregierung wie Bundestag auf deutliche Kritik ${ }^{45}$. Die Schaffung eines solchen „zweiten Parlaments“ wurde nicht nur als Konkurrenz gesehen, sondern schien auch der eigentlichen Aufgabe des Bundesrates, nämlich der Sicherung der Interessen der Länder in der Bundesgesetzgebung, nicht zu entsprechen. Insbesondere stand dessen Detailakribie der Forderung nach Art. 76 II GG entgegen, daß er innerhalb von drei Wochen zu einem ihm von der Bundesregierung zugeleiteten Gesetzentwurf Stellung nehmen mußte. Das Ansinnen des Bundesrates, diese Beratungsfrist zu verlängern, lehnte die Bundesregierung regelmäßig ab, um so den Zeitdruck aufrechtzuerhalten. Allerdings fand der Kanzler im Einzelfall Wege, um entsprechende Vorlagen noch

\footnotetext{
43 Vgl. Christoph Henzler, Fritz Schäffer 1945-1967 (München 1994) 318-333.

44 Vgl. BVerfGE 1, 144-162.

45 Vgl. hierzu Eilika Wunder, Hessen im Bundesrat. Zum föderalistischen Selbstverständnis der hessischen Landesregierung 1949-1955 (Wiesbaden 2000) 43-46.
} 
vor Verabschiedung im Kabinett dem Bundesrat zu übermitteln und ihn so in die Lage zu versetzen, seine Ausschußberatungen und die Beschlußfassung der Länderkabinette dann innerhalb der gesetzlich vorgegebenen Drei-Wochen-Frist abzuschließen. Gerade bei umfangreichen Gesetzesmaterialien oder politisch brisanten Vorhaben war ein solches Vorgehen unumgänglich.

Die Einflußmöglichkeiten der Länderkammer lagen vor allem im sogenannten ersten Durchgang, wenn die Bundesregierung die entsprechende Kabinettsvorlage zunächst dem Bundesrat zur Stellungnahme übersandte und anschließend dessen Abänderungsvorschläge zusammen mit ihrem Gesetzentwurf im Bundestag in erster Lesung einbrachte. In diesem ersten Durchgang konnte die Länderkammer ihre Ansichten, Interessen, Bedenken und Modifikationen artikulieren, die dann in die Beratungen des Bundestages und seine Ausschüsse einflossen. Im allgemeinen beriet der Bundesrat die ihm übersandte Kabinettsvorlage gründlich sowohl in seinem Plenum als auch in seinen Ausschüssen und formulierte detaillierte Abänderungsvorschläge. Der Bundestag konnte dann einschätzen, unter welchen Prämissen die Länderkammer später einem von ihm in dritter Lesung verabschiedeten Gesetzentwurf zustimmen würde bzw. wo entscheidende Hindernisse lagen, und konnte seine eigenen Beratungen hieran ausrichten. Wurden die Einwendungen der Länderkammer nicht hinreichend berücksichtigt, konnte sie im sogenannten zweiten Durchgang, also nach der Verabschiedung im Bundestag und der Zuleitung des so beschlossenen Gesetzes an den Bundesrat, Einspruch erheben oder - bei zustimmungspflichtigen Gesetzen - über ein Vermittlungsverfahren noch Modifikationen in ihrem Sinne durchsetzen. Angesichts dieses Beratungsprocedere geschah ein solcher Widerspruch eher selten und vor allem dann, wenn Länderinteressen betroffen waren. In der ersten Legislaturperiode wurde bei 545 im Bundestag verabschiedeten Gesetzen in 75 Fällen der Vermittlungsausschuß angerufen, in den folgenden Legislaturperioden ${ }^{46}$ verringerte sich dies sogar noch.

Von Anfang an war die parteienmäßige Politisierung des Bundesrates groß. Gewiß verstand er sich auch und in erster Linie als Vertreter der Bundesländer; in finanziellen Fragen oder in Kompetenzangelegenheiten war es das überragende Anliegen aller Landesregierungen, die gemeinsamen Interessen gegenüber dem Bund zu wahren, oder aber die Konfliktlinie verlief - wie beim Finanzausgleich $z$ wischen finanziell starken und schwachen Bundesländern. Dennoch zeigte sich sehr schnell, daß sowohl die Regierungsparteien als auch die Opposition im Bundestag versuchten, die ihnen politisch nahestehenden Landesregierungen auf ihre Seite zu ziehen und sie für ihre Anliegen einzuspannen. Insbesondere für die Sozialdemokraten war die Versuchung groß, in der Länderkammer durch SPDgeführte Landesregierungen ihre politischen Vorstellungen zur Geltung bringen zu können. In vielen Fällen konnte die größte Oppositionspartei auf diese Weise Mißerfolge im Parlament wettmachen und Gesetzgebungsvorhaben in ihrem

46 So die Angaben in Peter Schindler, Datenhandbuch zur Geschichte des Deutschen Bundestages 1949 bis 1999 (Baden-Baden 1999) 2450. Die Zahlen für die 2. WP sind 507/65, für die 3. WP 424/49, für die 4. WP 427/39 und für die 5. WP 453/39; ebd. 
Sinne beeinflussen. Vor allem Hessen und Hamburg erwiesen sich im Bundesrat als wichtige Bündnispartner der SPD-Fraktion. In dem Maße jedoch, in dem das Koalitionsmuster in den Ländern sich demjenigen auf Bundesebene anpaßte, die Bundesregierung somit im Bundesrat auf eine Stimmenmehrheit der unionsgeführten Landesregierungen setzen konnte, verringerten sich die Chancen der Opposition, über die Länderkammer im skizzierten Sinne Einfluß zu nehmen.

Das andere Hilfsmittel der Opposition wurde das Bundesverfassungsgericht. Aus den schon bald nach seiner Konstituierung ausbrechenden heftigen Auseinandersetzungen um seinen Status ${ }^{47}$ war es als Sieger hervorgegangen, so daß seine starke und autonome Stellung im Regierungssystem der Bundesrepublik und seine Möglichkeiten, Regierungshandeln zu kontrollieren, nicht mehr zurückgenommen werden konnten. Versuche, über die Wahl der Verfassungsrichter ${ }^{48}$ eine für die eigenen politischen Vorhaben günstige Zusammensetzung des Richterkollegiums zu erreichen, schlugen fehl. Zwar waren der Bundestag (durch einen Wahlmännerausschuß) sowie der Bundesrat die entsprechenden Wahlorgane, doch machte ein hohes Quorum (Zwei-Drittel-Mehrheit) es notwendig, sich im Vorfeld auf ein Personaltableau zu verständigen, das weder Regierungs- noch Oppositionsparteien benachteiligte. Zudem zeigten sich alle Verfassungsrichter wenig geneigt, im Sinne „ihrer" Wähler zu agieren.

Dennoch wurde die Verfassungsrechtsprechung in der Bundesrepublik zu einem erheblichen Teil von den Impulsen der politischen Opposition geprägt, war für sie doch die Versuchung groß, in Fällen, in denen sie ihre politischen Rechte durch Bundestagsmehrheit oder Bundesregierung beeinträchtigt sah, die Karlsruher Richter anzurufen. Dies konnte wenig spektakuläre Bereiche betreffen wie ein Urteilsspruch gegen eine von der Koalitionsmehrheit beschlossene Beschränkung der Redezeit in Plenardebatten ${ }^{49}$ oder die bereits erwähnte Klage gegen den Finanzvorbehalt der Bundesregierung in $\$ 96$ der Geschäftsordnung des Bundestages. Dies bezog sich aber auch auf markante Fälle wie die Organklage der SPDFraktion gegen das Petersberger Abkommen 1949 und den Kehler Hafenvertrag $1951^{50}$, in denen sie der Bundesregierung zur Last legte, die Ratifikation dieser Verträge durch den Bundestag versäumt und damit die Rechte des Parlaments übergangen zu haben. Dies betraf schließlich spektakuläre Verfahren wie die Normenkontrollanträge gegen den EVG-Vertrag 1952 und gegen das Saar-Abkommen 195551. Auch das Parteienrecht - Parteiengesetz und Parteienfinanzierung wurde in zentralen Punkten durch Urteile des Bundesverfassungsgerichts bestimmt.

47 Vgl. hierzu Heinz Laufer, Verfassungsgerichtsbarkeit und politischer Prozeß. Studien zum Bundesverfassungsgericht der Bundesrepublik Deutschland (Tübingen 1968) 254-334. 48 Vgl. hierzu ebd. 206-253.

49 Vgl. BVerfGE 10, 4. Vgl. hierzu Klaus Stüwe, Die Opposition im Bundestag und das Bundesverfassungsgericht (Baden-Baden 1997) 259-265.

$50 \mathrm{Vgl}$. hierzu ebd. 212-218.

51 Vgl. ebd. 219-224 und 234-244. 
Die hier skizzierte Konstellation bezieht sich vor allem auf die 1950er Jahre. Bereits in der dritten Legislaturperiode, als die Unionsparteien die absolute Mehrheit der Wählerstimmen wie der Bundestagsmandate gewannen und zusammen mit der Deutschen Partei über eine breite parlamentarische Mehrheit verfügten, mehrten sich Dissonanzen und Spannungen im Regierungslager. Die Autorität des Kanzlers, durch Präsidentschaftskrise und Fernsehstreit geschwächt, schwand bedenklich, sein fester Griff auf Fraktion wie Parlament begann, sich zu lockern. Zudem meldeten sich Vertreter der gesellschaftlichen Gruppen und Interessen lautstärker als zuvor zu Wort, was die Konsensfindung erschwerte. Die wechselvolle Geschichte des „Sozialpakets“, in dem die Lohnfortzahlung im Krankheitsfall, eine Krankenversicherungs- und eine Kindergeldreform kunstvoll zusammengeschnürt worden waren, mag für diese neue Situation stehen; der heftige parlamentarische Streit um die Ausgestaltung dieses Vorhabens durchzog die gesamten 1960er Jahre 52 , ohne daß ein Abschluß der Reformen gelang.

Die Koalition mit der FDP ab 1961 erwies sich als höchst konfliktreich; die Klagen über mangelnde Führung, fehlende Initiative und Konzeptionslosigkeit der Bundesregierung waren selbst in den Koalitionsfraktionen immer deutlicher zu hören. Die Profilierungsbemühungen des selbstbewußten kleineren Partners taten ein übriges, um Sand in das Getriebe der gemeinsamen Parlamentsarbeit zu streuen. Angesichts abnehmender wirtschaftlicher Wachstumsraten und schrumpfender Steuereinnahmen waren es vor allem Haushaltsprobleme, die immer wieder zu Differenzen um den wirtschafts- und sozialpolitischen Kurs führten. Die bisherigen Mechanismen der Konsensfindung funktionierten nicht mehr so reibungslos wie bisher, die Profilierungsbemühungen einiger CDU-Politiker um die Kanzlernachfolge brachten Unruhe in die Fraktion, und auch die politischen Vorstellungen zwischen älteren Fraktionsmitgliedern und parlamentarischen Neulingen differierten in wichtigen Punkten, so daß es für den Fraktionsvorsitzenden schwer war, eine einheitliche Linie herzustellen. Selbst ein politisch gewandter und energischer Koordinator wie Rainer Barzel ${ }^{53}$ konnte hier nur bedingt gegensteuern.

Der Schulterschluß in den eigenen Reihen war auch deshalb so schwierig, weil die politische Abgrenzung zur SPD löchriger wurde. Deren "Gemeinsamkeits“Kurs stellte bisher gewohnte parlamentarische Scheidelinien in Frage. In innenund sozialpolitischen Fragen pragmatischer als zuvor und bemüht, in das Wählerreservoir der bürgerlichen Parteien vorzustoßen, in der Außen- und Sicherheitspolitik sich ebenfalls dem Regierungskurs annähernd, bot sie immer weniger einen Gegenpart, der den Verbund des Koalitionslagers sicherstellte. Die parlamentarischen Verhältnisse waren unübersichtlicher geworden.

Allerdings gelang es dem Bundestag nicht, hierdurch größere politische Eigenständigkeit zu gewinnen. Trotz der Agonie von Adenauers Kanzlerschaft, trotz

52 Vgl. Die CDU/CSU-Fraktion im Deutschen Bundestag. Sitzungsprotokolle 1961-1966, bearb. v. Corinna Franz (Düsseldorf 2003) Einleitung 41-43.

$53 \mathrm{Vgl}$. zum Führungsstil Barzels ebd. 32-35. 
dessen Ansehensverlust in der „Spiegel“-Affäre, trotz der Festlegung auf ein Rücktrittsdatum zur Mitte der Legislaturperiode vermochte das Parlament am Rheinufer nicht, sich einen erweiterten Gestaltungsspielraum zu erkämpfen, und fand zu keinem markanteren Profil. Auch in der Kanzlerschaft Erhards änderte sich dies nicht, obwohl der personelle Wechsel im Palais Schaumburg auch im benachbarten Bundeshaus als Chance für einen politischen Neuanfang angesehen worden war. Klagen über mangelnde Unterrichtung und fehlende Konsultation durch die neue Bundesregierung, der sich verhärtende Konflikt zwischen sozialpolitischen Wünschen und fiskalpolitischen Zwängen und nicht zuletzt erneute Stellungskämpfe der potentiellen Nachfolger des neuen Regierungschefs, der es an Führungsqualitäten fehlen ließ, lähmten den Bundestag nach innen wie nach außen. Erst während der Großen Koalition, nun angeführt von dem Fraktionsvorsitzendenduo Rainer Barzel und Helmut Schmidt, konnte er sich deutlicher als eigenständiger Akteur hervortun.

Fragt man nun nach Rolle und Aktionsradius des Parlaments in der westdeutschen Kanzlerdemokratie, so fällt der Scheinwerferkegel zunächst einmal stärker auf den Regierungschef als auf den Bundestag. Bedingt durch das Grundgesetz, geprägt aber auch durch seine Amtsführung, war es Adenauer gelungen, die Kanzlerstellung zur gewichtigen und maßgebenden Machtposition im bundesrepublikanischen Regierungssystem in den 50er Jahren auszubauen. Im Kabinett hatte er sich als entscheidender Dirigent der Regierungspolitik etablieren können, mit dem Bundeskanzleramt stand ihm eine effiziente Koordinierungs- und Kontrollinstanz zur Verfügung, und auch die Regierungsfraktionen im Bundestag waren ihm alles in allem eine verläßliche Stütze. Der Kanzler, Personifizierung einer erfolgreichen Regierungspolitik, engagierter Parteistratege und Wahlkämpfer sowie Garant der wachsenden Souveränität und internationalen Anerkennung des westdeutschen Staates, war der Angelpunkt des bundesrepublikanischen Regierungssystems.

Das Parlament stand in seinem Schatten; durch die enge Bindung zwischen Regierung und Mehrheitsfraktionen und die - rhetorisch wie sachlich - scharfe Abgrenzung zur Opposition konnte es nur bedingt politische Eigenständigkeit gewinnen. Auch wenn Formen und Ausmaß der parlamentarischen Kooperation über Parteigrenzen hinweg größer waren als zunächst sichtbar - nach außen dominierte der Antagonismus. Der Opposition, also der SPD, stand kein wirklich scharfes Instrumentarium zur Seite, mit dem sie sich zum parlamentarischen Widerpart der Regierung und der sie stützenden Fraktionen hätte aufschwingen können; allerdings hat sie ihre Funktion, die Regierung zu kontrollieren, mit den ihr zu Gebote stehenden parlamentarischen Möglichkeiten durchaus genutzt, wobei im Einzelfall Bundesrat und Bundesverfassungsgericht mit zu Hilfe genommen wurden. Dennoch blieb der starke Kanzler das entscheidende Signum der 50er Jahre.

Gegen Ende dieses Jahrzehnts veränderten sich jedoch die Bauformen der Kanzlerdemokratie. Die Position des Regierungschefs verlor an Stärke, die Koalitionsfraktionen waren weniger eindeutig Stützen des Regierungskurses, zudem 
mangelte es ihnen deutlicher als zuvor an innerer Geschlossenheit, und auch das Parlament als Ganzes gewann neues Selbstbewußtsein. Erste Überlegungen ${ }^{54}$, die Außensicht des Bundestages und sein Ansehen in der Bevölkerung zu verbessern, die Plenarsitzungen interessanter und abwechslungsreicher zu gestalten, der Opposition größere Darstellungs- und Einflußchancen zuzugestehen und so den Parlamentarismus zu beleben, führten zu mehreren Änderungen des parlamentarischen Procedere. Vor allem gegen Ende der 60er Jahre verdichtete sich dies zu einer Fülle parlamentarischer Initiativen, mit denen die Rolle des Bundestages in der westdeutschen Verfassungsordnung neu vermessen werden sollte. Auch wenn die Reformen nur auf graduellen Wandel angelegt waren, stellten sie doch das neue Selbstverständnis des Parlaments als politischem Akteur im bundesdeutschen Regierungssystem heraus.

54 Vgl. hierzu Uwe Thaysen, Parlamentsreform in Theorie und Praxis (Opladen 1972), sowie Heinrich Oberreuter (Hrsg.), Parlamentsreform. Probleme und Perspektiven in westlichen Demokratien (Passau 1981). 